\title{
Image-based control relying on conic curves foliation for passing through a gate
}

\author{
Paolo Salaris, Christian Vassallo, Philippe Souères and Jean-Paul Laumond
}

\begin{abstract}
This paper presents a geometric approach to the problem of designing visual feedback control laws to steer a nonholonomic vehicle, equipped with a fixed monocular camera, through a gate. The originality of our approach is to introduce and exploit the natural geometry induced by the presence of a gate in the environment, e.g. bundle of hyperbolae, ellipses and circles, providing stabilizing feedback control laws that steer the vehicle through the middle of the gate. Moreover, using visual servoing we prove that this geometry can be measured directly in the camera image plane. As a consequence, we provide an image-based control scheme, avoiding the use of a state observer. Simulations in a realistic scenario and experiments are provided to show the effectiveness of our feedback control laws.
\end{abstract}

\section{INTRODUCTION}

This paper provides a solution to the problem of steering a nonholonomic vehicle through a gate or a door by using visual information coming from a fixed on-board camera. In mobile robotics, steering a vehicle towards a door, is a basic problem widely addressed in the literature. For example, in [1] door crossing is solved combining vision and ultrasonic sensor information. A similar problem has been solved in [2] for a large indoor surveillance robot equipped with a Kinect while crossing narrow doors. After detecting and locating the door, the robot is steered through it by a nonlinear adaptive controller. In [3] a solution to the crossing a door problem in unknown environment is proposed. The problem is solved by a dynamic path planning algorithm implementation based on successive frontier points determination and an adaptive tracking control law. A sensor based algorithm for guiding a wheelchair through a doorway has been proposed also in [4]. The controller uses a camera and a laser range finder to perform the navigation. The problem of limited Field-Of-View (FOV) constraints is also taken into account. In [5] the same problem is solved by dynamically generating Bézier-curve based trajectories, while in [6] the door crossing problem in unknown environment for a wheelchair has been solved by a dynamic path planning algorithm based on successive points determination.

The approach used in this paper is quite different from previous ones. Indeed, the main objective is to take benefits from the geometry that naturally emerges from the problem statement. In the plane of the robot motion, the door can be represented by two points: the feet of its vertical supports. The originality of our approach is to introduce coordinate systems relative to these two points. The plane around the door is hence foliated by using confocal (the points being

\footnotetext{
This work is supported by ERC Advanced Grant 340050 Actanthrope. Authors are with LAAS-CNRS, 7 avenue du colonel Roche, F-31400 Toulouse Cedex 4, France. e-mail: psalaris, cvassall, soueres, jplelaas.fr
}

the foci) hyperbolae and ellipses (a.k.a. elliptic coordinates system) and confocal circles that intersect at right angles (a.k.a. bipolar coordinates system). First, by using visual servoing, we show that there exists a direct link between these curves and the positions in the image plane of two visual landmarks located on the door supports and at the same height w.r.t. the plane of the robot motion. Second, we provide feedback control laws based on this geometry as well as proofs of asymptotic stability of the controlled system by using the Lasalle-Krasowskii principle. As both coordinates systems are immediately available in the image plane, we basically provide a so called Image-Based control scheme (see [7], [8]). As a consequence, neither a state observer nor other sensors, apart from the camera, are necessary to execute our visual servo control. As a consequence, by using elliptic and bipolar coordinates, the vehicle is able to localize itself w.r.t. the door.

Visual servoing techniques are often used both to drive a robot towards a target or with respect to it, with and without obstacles. For instance, in [9] two control strategies based on measurements coming from a pan camera and a 2D laser range sensor for steering the vehicle towards a target amidst obstacles has been provided. To avoid target occlusions, the obstacles are assumed to be smaller w.r.t. the target height. In [10] a landmark-based navigation approach among obstacles has been developed for humanoid robots. It integrates high-level motion planning capabilities and a stack of feasible visual servoing tasks based on footprints following. The motion planning is based on the shortest path synthesis provided in [11] where the limited FOV problem is taken into account. The FOV is also considered in [12] to reach a surface in the state space from which the robot can easily move towards the desired position by a straight line. In [13], to steer a vehicle toward a target, a robust control strategy w.r.t. uncertainty on the depth of the target points and that takes into account the limits of actuator dynamics and the visibility constraint has been provided.

\section{Problem Statement}

Let us consider a vehicle moving on a plane where a right-handed reference frame $\langle W\rangle$ is defined with origin in $O_{w}$ and axes $X_{w}, Z_{w}$. The configuration of the vehicle is described by $q(t)=(x(t), z(t), \theta(t))$, where $(x(t), z(t))$ is the position in $\langle W\rangle$ of a reference point of the vehicle, and $\theta(t)$ is the vehicle heading with respect to the $X_{w}$ axis. Using that notation and denoting $v(t)$ and $\omega(t)$ the forward and angular velocities, respectively, the kinematics is

$$
\left[\begin{array}{c}
\dot{x} \\
\dot{z} \\
\dot{\theta}
\end{array}\right]=\left[\begin{array}{cc}
\cos \theta & 0 \\
\sin \theta & 0 \\
0 & 1
\end{array}\right]\left[\begin{array}{c}
v \\
\omega
\end{array}\right] \text {. }
$$


The vehicle is equipped with a rigidly fixed pinhole camera with reference frame $\langle C\rangle=\left\{O_{c}, X_{c}, Y_{c}, Z_{c}\right\}$ such that the optical center $O_{c}$ corresponds to the robot's center $[x(t), z(t)]^{T}$ and the optical axis $Z_{c}$ is aligned with the robot's forward direction (see Fig. 1).

The main objective of this paper is to steer this nonholonomic vehicle through a door by using measurements coming from the on-board camera. The door is represented by two visual landmarks that are located on its right and left supports and denoted by $F^{L}$ and $F^{R}$ (superscripts $R$ and $L$ indicate the "Right" and "Left" supports of the door). We also assume that they are at equal height $h_{w}$ w.r.t. the plane of the robot motion. Without loss of generality, we assume that the cartesian coordinates of these two points in $\langle W\rangle$ are $F^{R}=\left(0, h_{w}, a\right)$ and $F^{L}=\left(0, h_{w},-a\right)$, respectively.

Based on the pinhole camera model [14], the position of the landmark in the image plane is given by

$$
{ }^{I} x_{i}=\alpha_{x} \frac{{ }^{c} x_{i}}{{ }^{c} z_{i}}, \quad{ }^{I} y_{i}=\alpha_{y} \frac{h}{{ }^{z_{i}}},
$$

with $i=\{R, L\}$ and where ${ }^{c} x$ and ${ }^{c} z$ are the coordinates of the landmark in the camera frame $\langle C\rangle, h$ is the height of the landmark from the plane $X_{c} \times Z_{c}$, while $\alpha_{x}$ and $\alpha y$ are the camera intrinsic parameters, achievable by a calibration procedure and representing the focal length of the camera in terms of pixel dimensions in the $x$ and $y$ direction respectively.

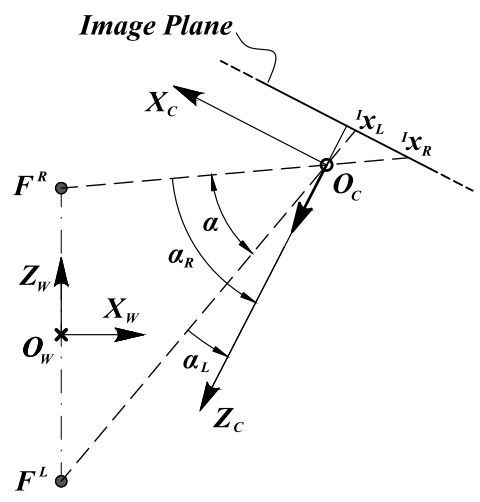

Fig. 1. Objective: to steer a vehicle through a door using only visual measurements. The door is represented by two landmarks, $F^{L}$ and $F^{R}$ and the vehicle, represented as a directed point, has an on-board camera and is subject to nonholonomic constraints.

Remark 1: We assume a camera with a large FOV (as human beings) so that the problem of keeping the landmarks in view is alleviated. This assumption does not impact the use of the control laws provided in next sections on a real system where the camera has a limited FOV.

\section{Some Basic Geometry Around The Door}

In this section we describe the intrinsic geometry that naturally emerges around the door and we show how this geometry is useful to design a feedback control law that steers the vehicle through it.

Referring to Fig. 1, let us assume that the forward velocity of the vehicle is constant, e.g. $v=1$, and the angular velocity is such that ${ }^{I} x_{R}(t) \equiv-{ }^{I} x_{L}(t)$ for all $t \in[0, T]$. In this case the vehicle is aligned with the bisector of angle $\widehat{F^{L} O_{c} F}$. In other words, the bearing angles ${ }^{1} \alpha_{R}(t)$ and $\alpha_{L}(t)$ w.r.t. each landmark have equal values but opposite signs. It is a well known fact that, by moving in this way, the vehicle follows a hyperbola, i.e. the locus of points where the absolute value of the difference between distances to the two foci (i.e. the projections on the motion plane of the two landmarks), is constant.

The parametric equations of a generic hyperbola with foci $F^{R}$ and $F^{L}$ are given by $x=a \cos \eta \sinh \xi, \quad z=a \sin \eta \cosh \xi$ with $\xi \in[0, \infty]$ and $\eta$ is constant with values in $[-\pi, \pi)$. In the canonical form we have $\frac{z^{2}}{a^{2} \sin ^{2} \eta}-\frac{x^{2}}{a^{2} \cos ^{2} \eta}=\cosh ^{2} \xi-$ $\sinh ^{2} \xi=1$. Hence, curves with constant $\eta$ form hyperbolae. In the special case of $\eta \in\{0, \pm \pi\}$, the hyperbola degenerates into a straight line passing perpendicularly through the middle of the segment between $F^{R}$ and $F^{L}$ (see Fig. 2). Let us now consider the case in which $\xi$ is constant and $\eta$ varies. Such curves, which are known as ellipses, can be expressed in the canonical form as $\frac{x^{2}}{a^{2} \sinh ^{2} \xi}+\frac{z^{2}}{a^{2} \cosh ^{2} \xi}=$ $\cos ^{2} \eta+\sin ^{2} \eta=1$.

The bundle of hyperbolae, obtained for different values of $\eta$ and the bundle of ellipses, obtained for different values of $\xi$, form an orthogonal coordinate system, a.k.a. elliptic coordinates, in which the coordinate lines are confocal ellipses and hyperbolae.

\section{A. Elliptic Coordinates}

Denoting by $\rho_{R}=\sqrt{(z-a)^{2}+x^{2}}$ and $\rho_{L}=\sqrt{(z+a)^{2}+x^{2}}$ the distance from the foci, i.e. the projections on the motion plane of the landmarks $F^{R}$ and $F^{L}$, respectively, the elliptic coordinates $(\xi, \eta)$ can be expressed as

$$
\begin{aligned}
& \xi=\operatorname{arccosh}\left(\frac{\rho_{R}+\rho_{L}}{2 a}\right) \\
& \eta=\frac{\pi}{2}-\arccos \left(\frac{\rho_{L}-\rho_{R}}{2 a}\right)
\end{aligned}
$$

Notice that, at the limit $a \rightarrow 0$, elliptic coordinates reduce to polar coordinates $(\rho, \psi)$. In particular, $\eta \rightarrow \psi$ and $a \cosh \xi \rightarrow \rho$. This also happens when the vehicle is sufficiently far from the door.

To complete this set of coordinates and to univocally describe the vehicle configurations, let us introduce the angle between the heading of the vehicle and the tangent to the hyperbola passing through the vehicle position:

$$
\beta_{e}=\arctan (\tanh \xi \tan \eta)-\theta+\pi .
$$

Notice that, $\beta_{e}$ becomes the bearing angle if $a \rightarrow 0$. Moreover, assuming a calibrated camera, it is possible to determine the elliptic coordinates directly by measurements of features in the image plane even if the height $h$ is unknown. The explicit expressions of the elliptic coordinates as functions of image measurements only are not reported for the sake of space. However, they can be simply obtained by computing the distances $\rho_{R}$ and $\rho_{L}$ of the landmarks from the vehicle and the distance $a$ between the landmarks w.r.t. the reference frame $\langle C\rangle$ by using (2) and then by substituting

\footnotetext{
${ }^{1}$ The bearing angle w.r.t. a target is the angle between the heading of the robot and the direction to the target.
} 


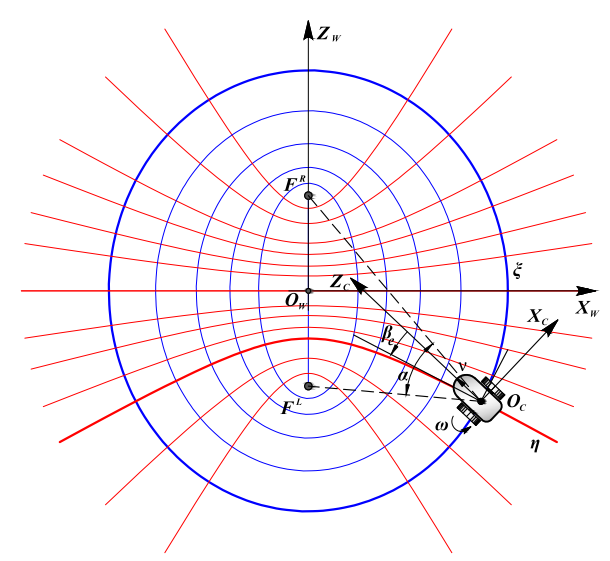

Fig. 2. Elliptic coordinate system. Ellipses and hyperbolae intersect perpendicularly.

them in (3), (4) and (5). The resulting expressions do not depend on $h$ and hence the control laws developed in the next section does not require a state observer, meaning that the robot is able to localize itself w.r.t. the door by using elliptic coordinates. In case of landmarks at different height, the elliptic coordinates also depend on these values that, being constants, can be considered known or estimated by an observer.

\section{B. Kinematic Model of the vehicle in Elliptic Coordinates}

The vehicle kinematic model in elliptic coordinates $\zeta=$ $\left(\xi, \eta, \beta_{e}\right)$, with $\xi \in[0, \infty]$ and $\eta \in(-\pi / 2, \pi / 2)$ is

$$
\begin{aligned}
\dot{\xi} & =-\frac{v \cos \beta \sec \eta \operatorname{sech} \xi}{a \sqrt{1+\tan ^{2} \eta \tanh ^{2} \xi}} \\
\dot{\eta} & =\frac{2 v \cos \eta \cosh \xi(\sin \gamma+\cos \gamma \tan \eta \tanh \xi)}{a(\cos (2 \eta)+\cosh (2 \xi))} \\
\dot{\beta}_{e} & =-\omega-\frac{2 v(\cos \beta \operatorname{sech} \xi \sin \eta-\sin \beta \sec \eta \sinh \xi)}{a(\cos (2 \eta)+\cosh (2 \xi)) \sqrt{1+\tan ^{2} \eta \tanh ^{2} \xi}}
\end{aligned}
$$

and $\gamma=\beta_{e}-\arctan (\tan \eta \tanh \xi)$.

Next section is dedicated to the design of a feedback control law that steers the vehicle through the middle of the door by exploiting the planar geometry that has been previously described.

\section{Feedback Control LaW in Elliptic COORDINATES}

Let us consider the problem of designing a feedback control law that steers the vehicle through the door as close as possible to the middle. To design a such control law, let us first consider the following change of inputs

$$
\begin{aligned}
& v=w \frac{a \sqrt{1+\tan ^{2} \eta \tanh ^{2} \xi}}{\sec \eta \operatorname{sech} \xi} \\
& \omega=-\omega_{o}+\frac{2 v(\cos \beta \operatorname{sech} \xi \sin \eta-\sin \beta \sec \eta \sinh \xi)}{a(\cos (2 \eta)+\cosh (2 \xi)) \sqrt{1+\tan ^{2} \eta \tanh ^{2} \xi}}
\end{aligned}
$$

where $w$ and $\omega_{o}$ are new control variables. By substituting (7) and (8) in (6) the kinematic model reduces to

$$
\begin{aligned}
\dot{\xi} & =-w \cos \beta_{e} \\
\dot{\eta} & =w \sin \beta_{e} \\
\dot{\beta}_{e} & =\omega_{o} .
\end{aligned}
$$

The objective is now to design $w$ and $\omega_{o}$ such that $\eta$ and $\beta_{e}$ converge to zero. Let us hence assume $w=\bar{w}$ and consider the following candidate of Lyapunov

$$
V\left(\eta, \beta_{e}\right)=\frac{1}{2}\left(\lambda \eta^{2}+\beta_{e}^{2}\right)
$$

where $\lambda$ is a positive constant parameter. Its time derivative, after substituting (9), becomes $\dot{V}\left(\eta, \beta_{e}\right)=\bar{w} \lambda \eta \sin \beta_{e}+$ $\beta_{e} \omega_{o}$, and by choosing

$$
\omega_{o}=-K \beta_{e}-\bar{w} \lambda \eta \frac{\sin \beta_{e}}{\beta_{e}}
$$

with $K>0$ a constant parameter, we obtain $\dot{V}\left(\eta, \beta_{e}\right)=$ $-K \beta_{e}^{2}$, that is negative semi-definite. However, the control $\omega_{o}$ is well definite and smooth everywhere. Let us define $R=$ $\left\{\left(\eta, \beta_{e}\right) \mid \dot{V}=0\right\}:$ in this case, we have that $R=\left\{\left(\eta, \beta_{e}\right) \mid \beta_{e}=\right.$ $0\}$. It is straightforward to observe that the only trajectory of (9) with (10) in $R$ is such that $\dot{\beta}_{e}=\omega_{o}=-\bar{w} \lambda \eta=0$. Hence, if $\bar{w}$ and $\lambda$ are not zero, $R$ does not contain any trajectory of the system, except the trivial trajectory $\left(\eta, \beta_{e}\right)=$ 0 . As a consequence all conditions of the local KrasowskiiLasalle principle are satisfied. We hence conclude that every trajectory starting from inside a given level curve of $V$ that contains the origin, converges to the origin as $t \rightarrow \infty$. Moreover, as $V$ is radially unbounded, we can conclude on the global asymptotic stability of the origin.

However, the control law (10), basically solves a path following problem in elliptic coordinates. A similar solution can be obtained in Cartesian coordinates to stabilize the vehicle along the $Z_{w}$ axis (models (1) and (9) are very similar). However, elliptic coordinates have some advantages: they can be obtained directly from measurements in the image plane (hence basically a state observer is not required) meaning that the vehicle can localize itself w.r.t. the door. Moreover, all hyperbolae pass through the door, hence guaranteeing that the vehicle, starting from an appropriate configuration, passes through it.

\section{THE BUNDLE OF CIRCLES}

The feedback control law provided in the previous section is not able to steer the vehicle through the middle of the door but only near to it. The distance between the middle of the door and the point where the vehicle crosses the door depends on both initial conditions and the values of the constant parameters $K$ and $\lambda$ in (10).

In this section, we will provide a feedback control law able to drive the vehicle exactly to the middle of the door. In order to do that, we will start analyzing the angle between the directions towards the two landmarks (angle $\alpha$ in Fig. 3) and its first time derivative. Then, we will show how this study brings to a particular geometry, i.e. two mutually orthogonal bundles of circles, that can be exploited to solve 
the problem at hand, overcoming all drawbacks of the control law furnished in the previous section.

Let us start recalling that for any point $O_{c}=(x, z)$ there always exists a circle $C_{\alpha}$ passing through $O_{c}$ and the projections of landmarks $F^{L}$ and $F^{R}$ on the motion plane. Angle $\alpha={\widehat{F^{L} O_{c} F}}^{R}$ is constant along $C_{\alpha}$ (a.k.a. angle at the circumference). Of course, on the contrary, for each value $\alpha \in[0, \pi]$ there are two circles passing through $F^{R}$ and $F^{L}$ and symmetric w.r.t. the $Z_{w}$ axis whose angle at the circumference is $\alpha$ : by varying $\alpha \in[0, \pi]$ we obtain a bundle of circles $\mathscr{C}_{\alpha}$ that, with the previous defined bundle of hyperbolae, generates a skew coordinates system. Indeed, circles and hyperbolae do not intersect orthogonally.

The expression of $\alpha$ in terms of $\xi$ and $\eta$ is

$$
\alpha=\arccos \left(1-\frac{4 \cos ^{2} \eta}{\cos (2 \eta)+\cosh (2 \xi)}\right) .
$$

while its time derivative $\dot{\alpha}$, which is not reported here for the sake of space, assumes the maximum value when

$$
\beta_{e}=\beta_{\text {max }}=-\operatorname{sgn}(\eta) \arccos \left(\frac{\sqrt{2} \cos \eta \cosh \xi}{\sqrt{\cos (2 \eta)+\cosh (2 \xi)}}\right),
$$

hence necessarily $\beta_{e} \neq 0$. Indeed, if $\beta_{e} \equiv \beta_{\max }$ the vehicle is aligned to the perpendicular to the circle $C_{\alpha}$ passing through the current vehicle position. Of course, this happens for all values of $\alpha$ and for all points in $C_{\alpha}$. It is well known that the set of all possible curves orthogonal to all members of $\mathscr{C}_{\alpha}$ constitutes a second bundle of circles $\mathscr{C}_{\alpha}^{\perp}$, as shown in Fig. 3 . In other words, for any point $Q \in C_{\alpha}$ there always exists a circle $C_{\alpha}^{\perp}$ of $\mathscr{C}_{\alpha}^{\perp}$, perpendicular to $C_{\alpha}$ in $Q$. Moreover, $C_{\alpha}^{\perp}$ crosses perpendicularly all circles of $\mathscr{C}_{\alpha}$.

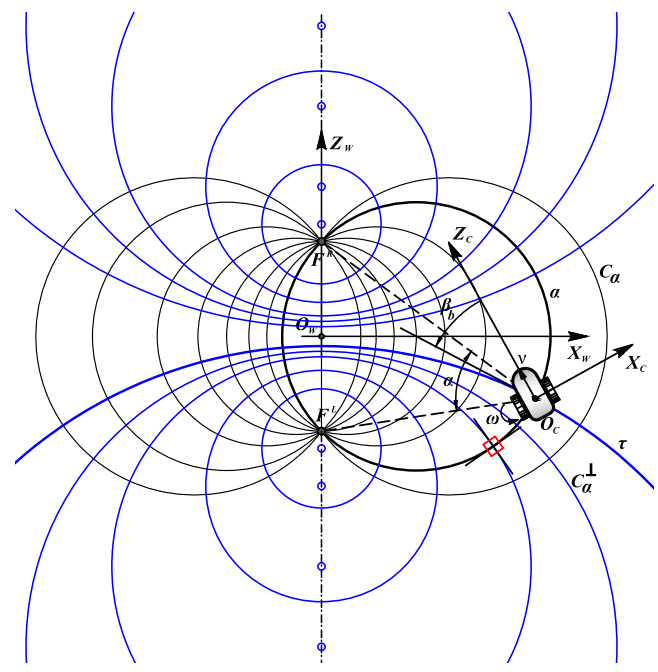

Fig. 3. Bipolar coordinate system. Circles intersect perpendicularly. Moreover, any circle of the bundle $C_{\alpha}$ is characterized by a constant angle (named $\alpha$ ), which is the angle at the circumference.

\section{A. Bipolar Coordinates}

The orthogonal bundles of circles previously introduced can be regarded as an orthogonal coordinates system also known as bipolar coordinates. The relationships between bipolar coordinates $\tau$ and $\alpha$ and the Cartesian coordinates $x$ and $z$ are given as

$$
x=\frac{a \sin \alpha}{\cosh \tau-\cos \alpha}, \quad z=\frac{a \sinh \tau}{\cosh \tau-\cos \alpha},
$$

assuming poles $F^{R}$ and $F^{L}$ on the $Z_{w}$ axis ( $2 a$ represents the distance between poles). Moreover, $\tau$ and $\alpha$ assume values in the following ranges $-\infty \leq \tau \leq \infty$ and $0 \leq \alpha \leq \pi$.

By denoting with $\rho_{R}=\sqrt{(z-a)^{2}+x^{2}}$ and $\rho_{L}=$ $\sqrt{(z+a)^{2}+x^{2}}$ the distance from the foci, i.e. the projections on the motion plane of landmarks $F^{R}$ and $F^{L}$, respectively, we have $\tau=\log \left(\frac{\rho_{L}}{\rho_{R}}\right)$ and $\alpha=\arccos \left(\frac{\rho_{R}^{2}+\rho_{L}^{2}-4 a^{2}}{2 \rho_{R} \rho_{L}}\right)$. From previous equations, after some algebra, it is possible to show that curves with constant $\tau$ are given by $x^{2}+\left(z-\frac{a}{\tanh \tau}\right)^{2}=$ $\frac{a^{2}}{\sinh ^{2} \tau}$, which is the equation of a circle whose center is on the $z$ axis with coordinates $\left(0, \frac{a}{\sinh \tau}\right)$ and radius $R=\frac{a}{|\sinh \tau|}$. These circles have been previously denoted by $C_{\alpha}^{\perp} \in \mathscr{C}_{\alpha}^{\perp}$. On the other hand, if $\alpha$ is constant, we obtain curves given by $\left(x-\frac{a}{\tan \alpha}\right)^{2}+z^{2}=\frac{a^{2}}{\sin ^{2} \alpha}$, which is the equation of a circle passing through the projection in the motion plane of landmarks $F^{R}$ and $F^{L}$, centered on the $X_{w}$ axis at $\left(\frac{a}{\tan \alpha}, 0\right)$ and radius $R=\frac{a}{|\sin \alpha|}$. These circles have been previously denoted by $C_{\alpha} \in \mathscr{C}_{\alpha}$.

To describe the position of the vehicle on the motion plane w.r.t. the door, let us consider a slightly different pair of coordinates, i.e. $\tau$ and $\hat{\alpha}=\pi-\alpha$. Notice that, $\hat{\alpha}$ is the supplementary angle of the angle at the circumference. Moreover, the middle of the door is the origin of those coordinates. Finally, as for elliptic coordinates, to univocally describe the vehicle configuration, let us introduce the angle $\beta_{b}$ between the heading of the vehicle and the tangent to the circle $C_{\alpha}^{\perp}$ passing through the vehicle position. The expression of this angle w.r.t. $\alpha$ and $\tau$ and $\theta$ is

$$
\beta_{b}=\arctan \left(\frac{\sin \alpha \sinh \tau}{1-\cos \alpha \cosh \tau}\right)-\theta+\pi .
$$

Notice that, as well as in case of the elliptic coordinates, also the bipolar coordinates can be computed directly from measurements of features in the image plane. The explicit expressions are not reported for space limitations but can be determined by following a similar procedure.

\section{B. Kinematic model of the vehicle in bipolar coordinates}

The kinematic model of the vehicle in bipolar coordinates $\lambda=\left(\tau, \hat{\alpha}, \beta_{b}\right)$ becomes

$$
\begin{aligned}
\dot{\tau} & =\frac{v}{a}\left((1+\cos \hat{\alpha} \cosh \tau) \sin \left(\beta_{b}-\operatorname{arccot}(\cot \hat{\alpha} \operatorname{coth} \tau+\csc \hat{\alpha} \operatorname{csch} \tau)\right)+\right. \\
& \left.+\cos \left(\beta_{b}-\operatorname{arccot}(\cot \hat{\alpha} \operatorname{coth} \tau+\csc \hat{\alpha} \operatorname{csch} \tau)\right) \sin \hat{\alpha} \sinh \tau\right) \\
\dot{\hat{\alpha}} & =\frac{v}{a}\left(-\cos \left(\beta_{b}-\operatorname{arccot}(\cot \hat{\alpha} \operatorname{coth} \tau+\csc \hat{\alpha} \operatorname{csch} \tau)\right)(\cos \hat{\alpha} \cosh \tau+1)+\right. \\
& \left.+\sin \hat{\alpha} \sin \left(\beta_{b}-\operatorname{arccot}(\cot \hat{\alpha} \operatorname{coth} \tau+\csc \hat{\alpha} \operatorname{csch} \tau)\right) \sinh \tau\right) \\
\dot{\beta}_{b} & =-\omega+\frac{v}{a} \frac{(\cos \hat{\alpha}+\cosh \tau)^{2}\left(\cos \beta_{b} \csc \hat{\alpha}+\operatorname{csch} \tau \sin \beta_{b}\right)}{(\cos \hat{\alpha}-\cosh \tau) \sqrt{1+(\cot \hat{\alpha} \operatorname{coth} \tau+\csc \hat{\alpha} \operatorname{csch} \tau)^{2}}}
\end{aligned}
$$

Recalling that the maximum value $\dot{\hat{\alpha}}_{\text {max }}$ of $\dot{\hat{\alpha}}$ is obtained along circles of $\mathscr{C}_{\alpha}^{\perp}$, i.e. with $\beta_{b}=0$, by the second equation of (14) we have

$$
\dot{\hat{\alpha}}_{\text {max }}=\left.\dot{\hat{\alpha}}\right|_{\beta_{b}=0}=-\frac{v(\cos \hat{\alpha}+\cosh \tau)}{a} .
$$


For positive values of $v,(15)$ is negative and is zero if $\tau=0$ and $\hat{\alpha}=\pi$ (or $\alpha=0$ ), i.e. very far from the door.

\section{Feedback Control Law in Bipolar COORDINATES}

The main idea of designing the feedback control law in bipolar coordinates is to define a smooth vector field $E$ obtained by derivation of an appropriated potential function $F$. Let us hence consider the following function

$$
F(\tau, \hat{\alpha})=\frac{(-\cos \hat{\alpha}+K \cosh \tau)}{a},
$$

where $K$ is a positive constant. This function is always positive and has a global minimum at the middle of the door, i.e. with $\tau=0$ and $\hat{\alpha}=0$. Notice that, $F$ is very similar to (15). By applying the differential nabla-operator ([15]) to the scalar function $F(\tau, \hat{\alpha})$, we obtain the vector field

$$
E(\tau, \hat{\alpha})=\nabla F(\tau, \hat{\alpha})=\frac{\cosh \tau-\cos \hat{\alpha}}{a^{2}}\left[\begin{array}{c}
K \sinh \tau \\
-\sin \hat{\alpha}
\end{array}\right] .
$$

Fig. 4(a) shows level curves of $F(\tau, \hat{\alpha})$ as well as the vector field. Notice that, all flow lines converge toward point $(\tau, \hat{\alpha})=(0,0)$, that, in Cartesian coordinates, corresponds to the middle of the door. In Fig. 4(b), the same potential field and associated vector field in Cartesian coordinates is also reported.

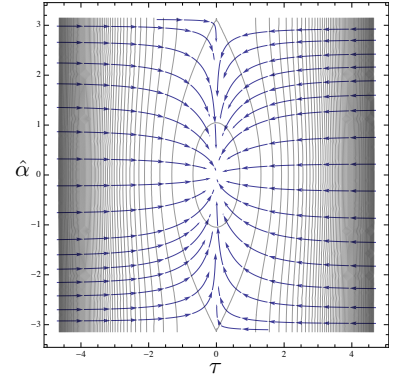
(a) Vector field in bipolar coordi- (b) Vector field in Cartesian coor-
nates.
dinates.

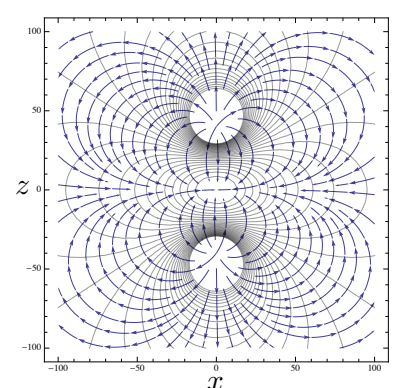

Fig. 4. Vector field obtained as the gradient of $F(\tau, \hat{\alpha})$ both in bipolar and Cartesian coordinates.

The objective is now to determine a feedback control law that steers the vehicle along the vector field represented in Fig. 4(a) in bipolar coordinates, or represented in Fig. 4(b) in Cartesian ones. Let $\phi$ be the angle $\beta_{b}$ when the vehicle is aligned with the vector field at any point $(\tau, \hat{\alpha})$. The angle $\phi$ can be easily obtained from (17), $\phi=$ $-\arctan 2(K \sinh \tau, \sin \hat{\alpha})$. Let us define the error $\sigma=\beta_{b}-\phi$ and force the dynamics of $s$ to be $\dot{\sigma}=-K_{\beta} \sigma, K_{\beta}>0$, by

$$
\begin{aligned}
\omega & =K_{\beta}\left(\beta_{b}-\phi\right)+\dot{\phi}+ \\
& +\frac{v}{a} \frac{(\cos \hat{\alpha}+\cosh \tau)^{2}\left(\cos \beta_{b} \csc \hat{\alpha}+\operatorname{csch} \tau \sin \beta_{b}\right)}{(\cos \hat{\alpha}-\cosh \tau) \sqrt{1+(\cot \hat{\alpha} \operatorname{coth} \tau+\csc \hat{\alpha} \operatorname{csch} \tau)^{2}}} .
\end{aligned}
$$

Once the vehicle is aligned with the vector field, it should reach the middle of the door. To do that, let us consider the following continuously differentiable function $V$ in terms of $\tau, \hat{\alpha}$ and $\sigma$,

$$
V=\frac{1}{2}\left(\tau^{2}+\hat{\alpha}^{2}+\sigma^{2}\right)
$$

and consider its time derivative along the trajectories of the system. By using (18) and choosing the forward velocity as

$$
v=K_{v}\left(\hat{\alpha} \cos \beta_{b}-\tau \sin \beta_{b}\right),
$$

we obtain

$\dot{V}=-K_{1}\left(\beta_{b}-\phi\right)^{2}-\frac{(\cos \hat{\alpha}+\cosh \tau)\left(\hat{\alpha} \cos \beta_{b}-\tau \sin \beta_{b}\right)^{2}}{a} \leq 0$,

which is negative semi-definite. As the function $V$ is positive definite, according to the Lasalle's invariance principle, the trajectories of the system converge to the largest invariant set $R=\{\lambda \mid \dot{V}(\lambda)=0\}$. By simple computation, $R=R_{1} \vee R_{2}$ where $R_{1}=\left\{\lambda \mid\left\{\hat{\alpha} \cos \beta_{b}-\tau \sin \beta_{b}=0\right\} \wedge\left\{\beta_{b}=\phi\right\}\right\}$ and $R_{2}=\left\{\lambda \mid\{\hat{\alpha}=0, \tau=0\} \wedge\left\{\beta_{e}=\phi\right\}\right\}$. After simple computations, we obtain that $R_{1}=\{\lambda \mid\{\hat{\alpha}=\pi\} \wedge\{\tau=0\}\}$ while $R_{2}=\{\lambda \mid \lambda=0\}$. It is possible to show that, if $K \neq 0, R_{1}$ is not an invariant set. Indeed, for $\lambda=[0, \pi, 0]^{T}$ we have $v \neq 0$ and thus the system can escape from $R_{1}$. As a consequence, point $\lambda=(0,0,0)$ is the only invariant set and we can conclude on the local asymptotic stability of the origin. Moreover, as the Lyapunov function is radially unbounded, we can conclude on the global asymptotic stability of the origin. The control law developed in this section is hence able to steer the vehicle exactly through the middle of the door.

\section{Simulations}

In this section, simulations showing the effectiveness of the control laws proposed in Sections IV and VI are presented. For both cases, two virtual frameworks are considered. In the first case, the robot is supposed to be inside a room and the objective is to leave the room passing through the door. In the second case, the robot is supposed to be in a corridor and the objective is to enter a room passing through the door. In both cases, the door is represented by two $3 \mathrm{D}$ points located in $(0,60,40) \mathrm{cm}$ and in $(0,60,-40) \mathrm{cm}$ w.r.t. a global reference frame. The 3D points of the scene are projected in the image plane through a virtual camera. The size of the image is $640 \times 480$ and the characteristic angle of the camera is almost $\pi$. Moreover, the image frames are captured with a frequency of 15 frames per second and different level of white gaussian noise with standard deviation $\sigma=5,10,15$ pixel to points in the image plane is also added. As in this paper the camera is fixed on the robot, it is not possible in the second scenario (see Figs. 5(d) and 6(d)) to perform the task without loosing at least one landmark, even if a very large Field-of-View has been considered. For this reason, in the second scenario we directly assume that elliptic or bipolar coordinates are available and some white Gaussian noise, equivalent to have a 5 pixel Gaussian noise in the image plane, is directly added to the state variables.

In Figs. 5 and 6 few trajectories of the vehicle are shown with and without noise for the two cases, chosen among the several trajectories analyzed for each case. The average error w.r.t. the middle of the door is also reported in the caption of each picture with and without noise $\left(\varepsilon_{w}\right.$ and $\varepsilon_{w o}$, respectively). The simulations show that both feedback control laws work properly in spite of image noise. However, the control law in bipolar coordinates seems to be more sensitive to image noise than the control law in elliptic 


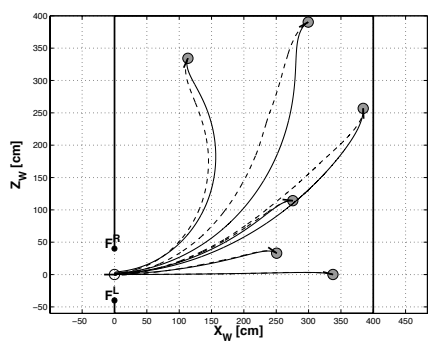

(a) $\sigma=5$ pixel: $\varepsilon_{w}=2.09+1.53 \mathrm{~cm}$ and $\varepsilon_{w o}=2.37 \pm 1.51 \mathrm{~cm}$.

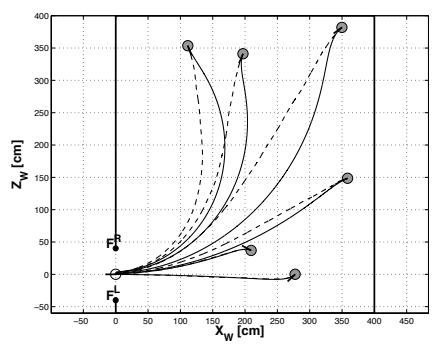

(b) $\sigma=10$ pixel: $\varepsilon_{w}=1.4$
and $\varepsilon_{w o}=2.37 \pm 1.43 \mathrm{~cm}$

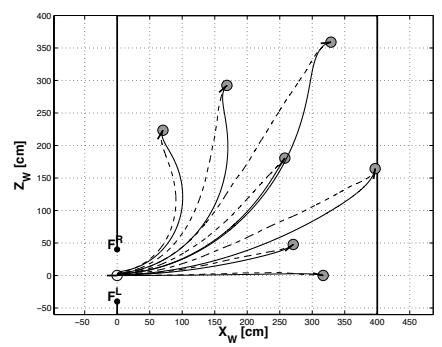

(c) $\sigma=15$ pixel: $\varepsilon_{w}=0.45 \pm 1.12 \mathrm{~cm}$ and $\varepsilon_{w o}=2.37 \pm 1.37 \mathrm{~cm}$.

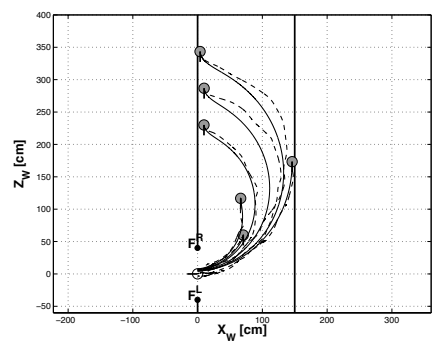

(d) $\sigma=5$ pixel: $\varepsilon_{w}=4.43 \pm 4.68 \mathrm{~cm}$ and $\varepsilon_{w o} \approx 6.85 \pm 2.92 \mathrm{~cm}$.

Fig. 5. Simulations with the feedback control law in elliptic coordinates: trajectories of the vehicle without and with white gaussian noise, continuous and dashed lines, respectively. The vehicle leaves a room in (a), (b) and (c) and enters a room from a corridor (d), by passing through a door.
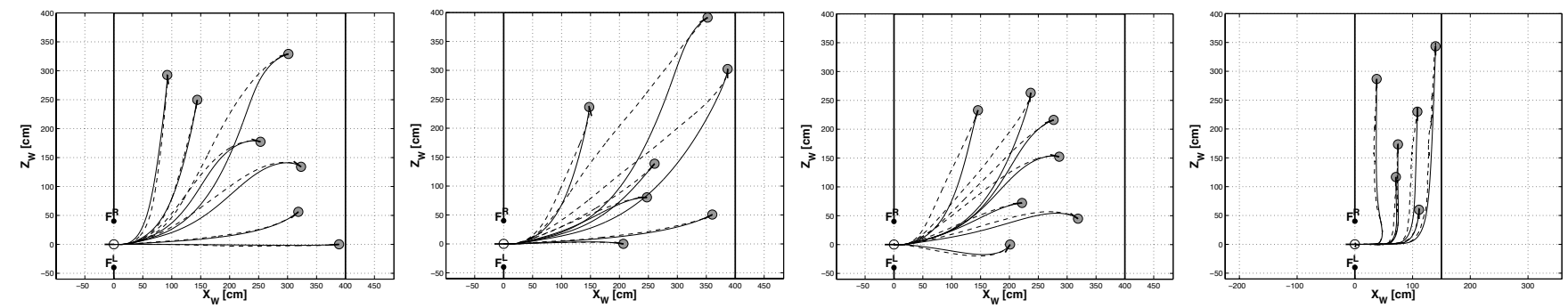

(a) $\sigma=5$ pixel: $\varepsilon_{w}=-0.027 \pm$ (b) $\sigma=10$ pixel: $\varepsilon_{w}=-0.043 \pm$ (c) $\sigma=15$ pixel: $\varepsilon_{w}=-0.053 \pm(\mathrm{d}) \sigma=5$ pixel: $\varepsilon_{w}=0.1 \pm 0.33 \mathrm{~cm}$ $0.016 \mathrm{~cm}$ and $\varepsilon_{w o}=-0.0113 \pm 0.028 \mathrm{~cm}$ and $\varepsilon_{w o}=-0.0095 \pm 0.040 \mathrm{~cm}$ and $\varepsilon_{w o}=-0.0095 \pm$ and $\varepsilon_{w o} \approx 0 \mathrm{~cm}$.
$0.0053 \mathrm{~cm}$.
$0.0036 \mathrm{~cm}$.
$0.0039 \mathrm{~cm}$

Fig. 6. Simulations with the feedback control law in bipolar coordinates: trajectories of the vehicle with and without white gaussian noise, continuous and dashed lines, respectively. The vehicle leaves a room in (a), (b) and (c) and enters a room from a corridor (d), by passing through a door.

coordinates when the vehicle is far away from the door, while it works very well near to the door, especially while entering a room from the corridor. This may suggest a possible strategy: use elliptic control laws when the robot is far away from the door and bipolar ones when it is sufficiently near to the door or when the vehicle is very near the wall. Future works will be dedicated to better investigate this strategy.

\section{EXPERIMENTS}

The experimental setup comprised of a Vivotek Wireless Network Camera with Pan-Tilt (PT7137) fixed at the middle of the wheel axel of the RobuLAB-10 mobile platform by Robosoft. The image resolution was $640 \times 480$ and the camera Horizontal and vertical FOV magnitude are around $50 \mathrm{deg}$ and $40 \mathrm{deg}$, respectively. Moreover, in our experiments, the pan-tilt mechanism is not used. The camera has been calibrated by using tools available in the Opencv vision library. The result of this procedure, which cannot be reported here for the sake of space, showed that the camera presented radial distortions. The noise level has been estimated to have a standard deviation $\sigma=0.05$ pixel. Moreover, the controller rate was around $20 \mathrm{~Hz}$.

In this paper we did not consider the problem of door detection. The door is indeed represented by two circular landmarks at the same height from the plane of the robot motion. The projection of the landmarks in the image plane are detected by using tools available in the Opencv and VISP libraries. Because of the limited FOV aperture, the robot loses the landmarks while passing through the door. In our experiments, the parameters in the control laws are chosen such that the vehicle is almost aligned to the middle of the door just before losing the landmarks. Starting from this configuration, which is quite close to the door (depending on the FOV magnitude), an open loop control law with $\omega=0$ and $v=$ const. is applied. Of course, other better solutions might be used. For example, assuming the height of the landmarks known and by using the pinhole camera model, starting from the last available positions of the landmarks in the image plane, a prediction of the future ones can be obtained and used in the control laws. In Figs. 7 and 8 the trajectories of the robot starting from different initial configurations and obtained by applying the feedback control laws based on elliptic and bipolar coordinates, respectively, are reported. The state variables of the robot and hence the trajectories towards the door have been measured by using the Motion Capture system. Of course, no information coming from this sensor are used in the control law which is a purely Image-Based visual servo control. Finally, the distance between the two landmarks is $68 \mathrm{~cm}$. A video of the experiments is available (Video).

\section{CONClusions And Future WORKS}

In this paper, a geometric approach to steer a robot subject to nonholonomic constraints through a door by using visual measurements coming from a fixed on-board monocular camera, has been provided. Feedback control laws able to steer the vehicle through the middle of the door by exploiting the natural planar geometry induced by the presence of a door in the environment have been obtained. Simulations and experimental results have been also reported. 


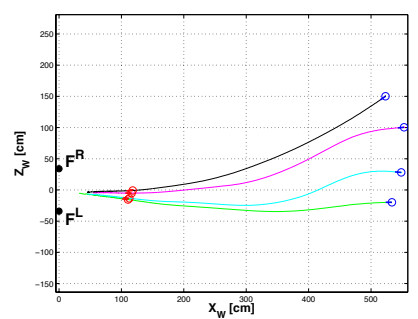

(a) Vehicle trajectories.

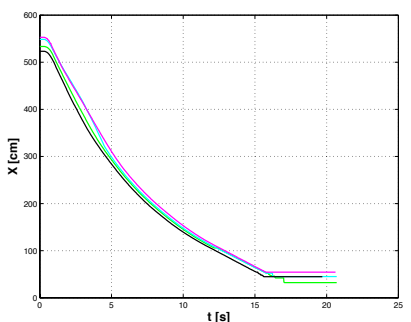

(b) $x$.

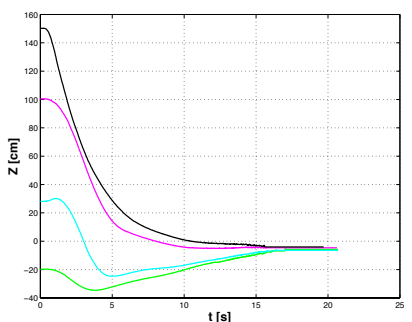

(c) $z$

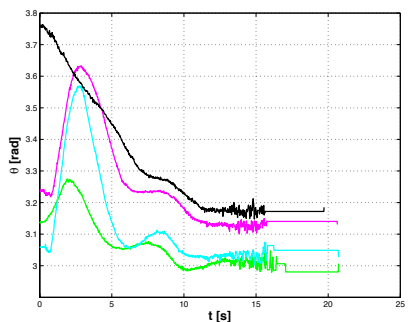

(d) $\theta$.

Fig. 7. Experimental results obtained applying the control law in elliptic coordinates. The control parameters are $w=0.0012, K=0.7$ and $\lambda=310$. Average linear velocity of $0.3 \mathrm{~m} / \mathrm{s}$.

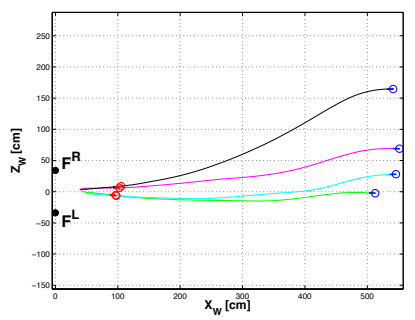

(a) Vehicle trajectories.

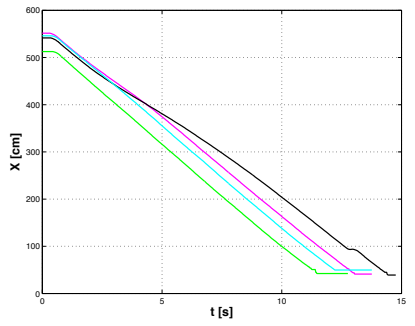

(b) $x$.

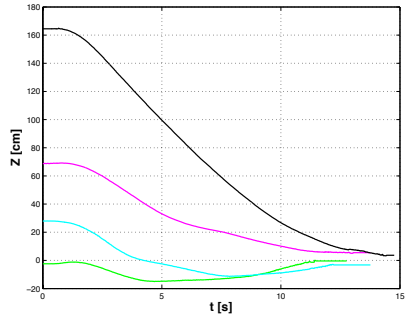

(c) $z$

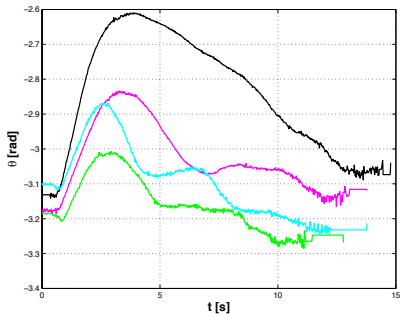

(d) $\theta$.

Fig. 8. Experimental results obtained applying the control law in bipolar coordinates. The control parameters are $K=2, K_{1}=4$ and $K_{V}=0.15$. Average linear velocity of $0.4 \mathrm{~m} / \mathrm{s}$

The objective of this paper has also been to furnish an interesting base for future studies on the investigation of human trajectories during a rest-to-rest task, i.e. from an initial configuration to a final one. Our ultimate goal is to characterize the role of sensory systems and of the geometry of the environment in the shape of the trajectories followed by human beings. From a biological point of view, a lot of effort has been done to investigate how sensory systems, and in particular vision, influence motion patterns of human beings while going towards a target [16]. In particular, when a human subject goes toward a target at a constant velocity, it has been shown that it behaves as a nonholonomic system: the velocity remains tangent to the sagittal plane. A fundamental step in this view is to introduce in the framework of this paper a pan-tilt mechanism to take into account that humans can turn the head. This is very important in order to accomplish the task of entering a room from a corridor. Future work will be also to extend the feedback control laws in presence of obstacles. Indeed, the two landmarks representing the door can be seen as the nearest points, in the image plane, between two obstacles.

\section{REFERENCES}

[1] I. Monasterio, E. Lazkano, I. Rañó, and B. Sierra, "Learning to traverse doors using visual information," Mathematics and Computers in Simulation, vol. 60, no. 3, pp. 347-356, 2002.

[2] A. Dev, B. Krose, and F. Groen, "Detecting, locating and crossing a door for a wide indoor surveillance robot," in IEEE International Conference on Robotics and Biomimetics (ROBIO)., Dec 2013, pp. $1740-1746$.

[3] F. Cheein, C. De La Cruz, T. Bastos, and R. Carelli, "Slam-based cross-a-door solution approach for a robotic wheelchair," Int. J. Adv. Robot Syst., vol. 7, no. 2, pp. 155-164, 2010.

[4] S. Patel, S.-H. Jung, J. Ostrowski, R. Rao, and C. Taylor, "Sensor based door navigation for a nonholonomic vehicle," in IEEE International
Conference on Robotics and Automation (ICRA)., vol. 3, 2002, pp. 3081-3086.

[5] S. Wang, L. Chen, H. Hu, and K. McDonald-Maier, "Doorway passing of an intelligent wheelchair by dynamically generating bèzier curve trajectory," in IEEE International Conference on Robotics and Biomimetics (ROBIO), Dec 2012, pp. 1206-1211.

[6] C. Fernando A. Auat, D. L. C. Celso, B. Teodiano F., and C. Ricardo, "Slam-based cross-a-door solution approach for a robotic wheelchair," International Journal of Advanced Robotic Systems, vol. 7, no. 2, pp. 155-164, 2010.

[7] F. Chaumette and S. Hutchinson, "Visual servo control, Part I: Basic approaches," IEEE Robotics and Automation Magazine, vol. 13, no. 4, pp. 82-90, December 2006.

[8] —, "Visual servo control, Part II: Advanced approaches," IEEE Robotics and Automation Magazine, vol. 14, no. 1, pp. 109-118, March 2007.

[9] D. Dedieu, V. Cadenat, and P. Souères, "Mixed camera-laser based control for mobile robot navigation," in IEEE/RSJ International Conference on Intelligent RObots and Systems, (IROS)., vol. 2, 2000, pp. $1081-1086$

[10] J.-B. Hayet, C. Esteves, G. Arechavaleta, O. Stasse, and E. Yoshida, "Humanoid locomotion planning for visually guided tasks," International Journal of Humanoid Robotics, vol. 09, no. 02, 2012.

[11] P. Salaris, D. Fontanelli, L. Pallottino, and A. Bicchi, "Shortest paths for a robot with nonholonomic and field-of-view constraints," IEEE Transactions on Robotics, vol. 26, no. 2, pp. 269-281, April 2010.

[12] N. Gans and S. Hutchinson, "A stable vision-based control scheme for nonholonomic vehicles to keep a landmark in the field of view," in Proc. IEEE Int. Conf. on Robotics and Automation, apr. 2007, pp. $2196-2201$.

[13] P. Souères, S. Tarbouriech, and B. Gao, "A robust vision-based controller for mobile robots navigation: application to the task sequencing problem," in IEEE/RSJ International Conference on Intelligent RObots and Systems (IROS), Aug 2005, pp. 2191-2196.

[14] R. Hartley and A. Zisserman, Multiple View Geometry in Computer Vision. Cambridge University Press, 2003.

[15] M. Cleve, "Mathematical handbook for scientists and engineers," Engineering and Science, vol. 25, no. 2, pp. 6-6, 1961.

[16] K. Mombaur, A. Truong, and J.-P. Laumond, "From human to humanoid locomotion-an inverse optimal control approach," Auton. Robots, vol. 28, no. 3, pp. 369-383, Apr 2010. 